\title{
The Infrared Behavior of the Gluon and Ghost Propagators in SU(2) Yang-Mills Theory in the Maximal Abelian Gauge
}

\author{
M.A.L. Capri ${ }^{a}$, D. Dudal ${ }^{b}$, J.A. Gracey ${ }^{c}$, V.E.R. Lemes $^{c}$, R.F. Sobreiro ${ }^{a}$, S.P. Sorella ${ }^{a}{ }^{*}$ R. Thibes ${ }^{a}$, and H. Verschelde ${ }^{a}$ \\ ${ }^{a}$ UERJ - Universidade do Estado do Rio de Janeiro \\ Rua São Francisco Xavier 524, 20550-013 Maracanã \\ Rio de Janeiro, Brazil \\ ${ }^{b}$ Ghent University \\ Department of Mathematical Physics and Astronomy \\ Krijgslaan 281-S9 \\ B-9000 Gent, Belgium \\ ${ }^{c}$ Theoretical Physics Division \\ Department of Mathematical Sciences \\ University of Liverpool \\ P.O. Box 147, Liverpool, L69 3BX, United Kingdom
}

Received on 6 August, 2006

\begin{abstract}
We report on some recent analytical results on the behaviour of the gluon and ghost propagators in Euclidean $S U$ (2) Yang-Mills theory quantized in the maximal Abelian gauge (MAG). This gauge is of particular interest for the dual superconductivity picture to explain color confinement. Two kinds of effects are taken into account: those arising from a treatment of Gribov copies in the MAG and those arising from a dynamical mass originating in a dimension two gluon condensate. The diagonal component of the gluon propagator displays the typical Gribov-type behaviour, while the off-diagonal component is of the Yukawa type due to the dynamical mass. These results are in qualitative agreement with available lattice data on the gluon propagators. The off-diagonal ghost propagator exhibits an infrared enhancement due to the Gribov restriction, while the diagonal one remains unaffected.
\end{abstract}

Keywords: Yang-Mills gauge theory; Maximal Abelian gauge; Faddeev-Popov operator; Propagators; Gribov-Zwanziger scenario; Condensates

\section{INTRODUCTION}

We shall consider pure Euclidean $S U(N)$ Yang-Mills theories with action

$$
S_{Y M}=\frac{1}{4} \int d^{4} x \mathcal{F}_{\mu \nu}^{a} \mathcal{F}_{\mu \nu}^{a}
$$

where $\mathcal{A}_{\mu}^{a}, a=1, \ldots, N^{2}-1$ is the gauge boson field, with associated field strength

$$
\mathcal{F}_{\mu \nu}^{a}=\partial_{\mu} \mathcal{A}_{v}^{a}-\partial_{\nu} \mathcal{A}_{\mu}^{a}+g f^{a b c} \mathcal{A}_{\mu}^{b} \mathcal{A}_{v}^{c}
$$

The theory (1) is invariant w.r.t. the local gauge transformations

$$
\delta \mathcal{A}_{\mu}^{a}=\mathcal{D}_{\mu}^{a b} \omega^{b}
$$

with

$$
\mathcal{D}_{\mu}^{a b}=\partial_{\mu} \delta^{a b}-g f^{a b c} \mathcal{A}_{\mu}^{c},
$$

denoting the adjoint covariant derivative.

\footnotetext{
*Work supported by FAPERJ, Fundação de Amparo à Pesquisa do Estado do Rio de Janeiro, under the program Cientista do Nosso Estado, E26/151.947/2004.
}

As it is well known, the theory (1) is asymptotically free $[1,2]$, i.e. the coupling becomes smaller at lower energies and vice versa. At very high energies, the interaction is weak and the gluons can be considered as almost free particles. However, in spite of the progress in the last decades, we still lack a satisfactory understanding of the behaviour of Yang-Mills theories in the low energy regime. Here the coupling constant of the theory is large and nonperturbative effects have to be taken into account. One of the greatest challenges of contemporary theoretical physics is to actually prove that (1) describes a confining theory in the infrared. Its physical spectrum is believed to be given by colorless bound states of gluons called glueballs. We adopt here the common paradigm according to which the first step for understanding color confinement in the real world, i.e. in the QCD-world, is to understand it first in pure Yang-Mills theory (1).

A partial list of causes of nonperturbative effects is given by Gribov ambiguities which affect the Faddeev-Popov quantization procedure and hence the propagators [3], the existence of condensates such as $\left\langle F^{2}\right\rangle,\left\langle A^{2}\right\rangle$, etc. [4-6], the existence of (topologically) nontrivial field configurations like instantons [7], etc. These effects are not necessarily unrelated, as e.g. instantons can contribute to the condensate $\left\langle F^{2}\right\rangle$ [7], the Faddeev-Popov operator has zero modes in an instanton background [8], etc. 


\section{A. The dual superconductivity picture}

A particularly appealing proposal to explain confinement in the low energy regime was made in [9-11]. Let us give a simple idea of the picture. If the QCD vacuum would contain magnetic monopoles and if these monopoles would condense, there will be a dual Meissner effect which squeezes the chromoelectric field into a thin flux tube. This results in a linearly rising potential, $V(r)=\sigma r$, between static charges, as can be guessed from Gauss' law, $\int E d S=$ const or, since the main contribution is coming from the flux tube, one finds $E \Delta S \approx$ const, hence $V=-\int E d r \propto r$ where $\Delta S$ is the area of the flux tube. In fact, it is not difficult to imagine the longer the flux tube (string) gets, the more energy it will carry. Hence, it would cost enormous amounts of energies to separate two test charges from each other and so they are confined to stay together.

\section{B. The maximal Abelian gauge}

An important ingredient for the dual superconductivity picture to work, is clearly the presence of (magnetic) monopoles. As the Yang-Mills action (1) is locally gauge invariant, we need to fix the gauge in order to quantize it. 't Hooft invented the so-called Abelian gauges in [12], of which the maximal Abelian gauge (MAG) is a specific example [12-14]. In these Abelian gauges, defects in the gauge fixing give rise to pointlike singularities which can be associated to monopoles.

We may decompose the $S U(2)$ gauge field into off-diagonal and diagonal components, according to

$$
\mathcal{A}_{\mu}=A_{\mu}^{a} T^{a}+A_{\mu} T^{3}
$$

where $T^{a}, a=1,2$, denote the off-diagonal generators of $S U$ (2), while $T^{3}$ stands for the diagonal generator,

$$
\left[T^{a}, T^{b}\right]=i \varepsilon^{a b} T^{3}, \quad\left[T^{3}, T^{a}\right]=i \varepsilon^{a b} T^{b}
$$

where

$$
\varepsilon^{a b}=\varepsilon^{a b 3}, \quad \varepsilon^{a c} \varepsilon^{a d}=\delta^{c d} .
$$

Similarly, for the field strength one shall find

$$
\mathcal{F}_{\mu v}=F_{\mu \nu}^{a} T^{a}+F_{\mu v} T^{3}
$$

with off-diagonal and diagonal parts given by

$$
\begin{aligned}
& F_{\mu \nu}^{a}=D_{\mu}^{a b} A_{v}^{b}-D_{v}^{a b} A_{\mu}^{b}, \\
& F_{\mu \nu}=\partial_{\mu} A_{v}-\partial_{v} A_{\mu}+g \varepsilon^{a b} A_{\mu}^{a} A_{v}^{b},
\end{aligned}
$$

The covariant derivative $D_{\mu}^{a b}$ is defined with respect to the diagonal component $A_{\mu}$

$$
D_{\mu}^{a b} \equiv \partial_{\mu} \delta^{a b}-g \varepsilon^{a b} A_{\mu}
$$

For the Yang-Mills action in Euclidean space, one finally obtains

$$
S_{\mathrm{YM}}=\frac{1}{4} \int d^{4} x\left(F_{\mu \nu}^{a} F_{\mu \nu}^{a}+F_{\mu \nu} F_{\mu \nu}\right) .
$$

As it is easily checked, the classical action (11) is left invariant by the decomposed gauge transformations

$$
\begin{aligned}
& \delta A_{\mu}^{a}=-D_{\mu}^{a b} \omega^{b}-g \varepsilon^{a b} A_{\mu}^{b} \omega, \\
& \delta A_{\mu}=-\partial_{\mu} \omega-g \varepsilon^{a b} A_{\mu}^{a} \omega^{b} .
\end{aligned}
$$

The MAG is obtained by demanding

$$
D_{\mu}^{a b} A_{\mu}^{b}=0,
$$

which follows by requiring that the auxiliary functional

$$
\mathcal{R}[A]=\int d^{4} x A_{\mu}^{a} A_{\mu}^{a},
$$

is stationary with respect to the gauge transformations (12).

Moreover, as it is apparent from the presence of the covariant derivative $D_{\mu}^{a b}$, (13) allows for a residual local $U(1)$ invariance corresponding to the diagonal subgroup of $S U(2)$. This additional invariance has to be fixed by means of a suitable gauge condition on the diagonal component $A_{\mu}$, which will be chosen to be of the Landau type, also adopted in lattice simulations [15, 16], namely

$$
\partial_{\mu} A_{\mu}=0
$$

The MAG is interesting from the lattice as well as continuum viewpoint. It can be simulated numerically, as first discussed in $[13,14]$, while the MAG is also renormalizable in the continuum. Although strictly speaking, the MAG as defined by (13) is not renormalizable. A slight generalization has to be considered containing a gauge parameter $\alpha$. Moreover, due to the nonlinearity of the gauge condition (13), a quartic ghost interaction has to introduced in the MAG action [17]. The complete action turns out to be given by

$$
S=S_{\mathrm{YM}}+S_{\mathrm{MAG}}+S_{\mathrm{diag}},
$$

where $S_{\mathrm{MAG}}, S_{\mathrm{diag}}$ are the gauge fixing terms corresponding to the off-diagonal and diagonal sectors, respectively, given by 


$$
\begin{aligned}
S_{\mathrm{MAG}} & =s \int d^{4} x\left(\bar{c}^{a}\left(D_{\mu}^{a b} A_{\mu}^{b}+\frac{\alpha}{2} b^{a}\right)-\frac{\alpha}{2} g \varepsilon^{a b} \bar{c}^{a} \bar{c}^{b} c\right) \\
& =\int d^{4} x\left(b^{a}\left(D_{\mu}^{a b} A_{\mu}^{b}+\frac{\alpha}{2} b^{a}\right)+\bar{c}^{a} D_{\mu}^{a b} D_{\mu}^{b c} c^{c}+g \bar{c}^{a} \varepsilon^{a b}\left(D_{\mu}^{b c} A_{\mu}^{c}\right) c-\alpha g \varepsilon^{a b} b^{a} \bar{c}^{b} c-g^{2} \varepsilon^{a b} \varepsilon^{c d} \bar{c}^{a} c^{d} A_{\mu}^{b} A_{\mu}^{c}-\frac{\alpha}{4} g^{2} \varepsilon^{a b} \varepsilon^{c d} \bar{c}^{a} \bar{c}^{b} c^{c} c^{d}\right) \\
S_{\mathrm{diag}} & =s \int d^{4} x \bar{c} \partial_{\mu} A_{\mu}=\int d^{4} x\left(b \partial_{\mu} A_{\mu}+\bar{c} \partial_{\mu}\left(\partial_{\mu} c+g \varepsilon^{a b} A_{\mu}^{a} c^{b}\right)\right)
\end{aligned}
$$

where $s$ is the nilpotent $\left(s^{2}=0\right)$ BRST transformation, defined through

$$
\begin{aligned}
s A_{\mu}^{a} & =-\left(D_{\mu}^{a b} c^{b}+g \varepsilon^{a b} A_{\mu}^{b} c\right) \\
s A_{\mu} & =-\left(\partial_{\mu} c+g \varepsilon^{a b} A_{\mu}^{a} c^{b}\right) \\
s c^{a} & =g \varepsilon^{a b} c^{b} c, \quad s c=\frac{g}{2} \varepsilon^{a b} c^{a} c^{b} \\
s \bar{c}^{a} & =b^{a}, \quad s \bar{c}=b, \\
s b^{a} & =0, \quad s b=0 \\
s S & =0 .
\end{aligned}
$$

$\left(\bar{c}^{a}, c^{a}\right)$ and $(\bar{c}, c)$ are the off-diagonal and diagonal FaddeevPopov ghosts, while $\left(b^{a}, b\right)$ denote the Lagrange multipliers. The action (16) is renormalizable to all orders of perturbation theory $[18,19]$. Only at the end, when the ultraviolet divergences are consistently treated and the theory is renormalized, can one consider the limit $\alpha \rightarrow 0$ which is formally equivalent with the condition (13). We refer to $[18,19]$ for details concerning the MAG renormalization.

\section{Abelian dominance}

According to the concept of Abelian dominance, the low energy regime of QCD can be expressed solely in terms of Abelian degrees of freedom [20]. Lattice confirmations of the Abelian dominance can be found in $[21,22]$. An argument that can be interpreted in favour of it, is the fact that the off-diagonal gluons would attain a large, dynamical mass. At energies below the scale set by this mass, the off-diagonal gluons should decouple, and in this way one should end up with an Abelian theory at low energies.

\section{Why study propagators?}

The reader might question why one would study the propagators, which are not even gauge invariant objects. Still, a certain number of considerations can be outlined. To some extent, propagators are the simplest Green's functions to be evaluated. Nowadays, it is possible to obtain analytic estimates of the influence of nonperturbative effects, like Gribov copies and condensates, on the infrared behaviour of the propagators. As far as $4 D$ Yang-Mills theory is considered, this task appears to be very difficult for more complicated Green's functions. In the last decade there has been an intensive activity from the lattice community in the study of the gluon and ghost propagators in a variety of gauges. We can thus compare our theoretical predictions with the available lattice data. So far, a certain number of gauges have been considered extensively from theoretical as well as from the lattice point of view. This is the case of the Landau, Coulomb and maximal Abelian gauge. In the following, we shall focus on the MAG. We shall see that the agreement between lattice results and theoretical investigations can be considered satisfactory.

The study of the propagators might also provide a useful framework to investigate the behaviour of the running coupling constant in the infrared. This is the case, for example, of the Landau gauge, for which the following relation holds

$$
Z_{g}=Z_{A}^{-1 / 2} Z_{c}^{-1}
$$

to all orders of perturbation theory (see e.g. [23]). If this relation is expected to be valid at the nonperturbative level, the infrared behavior of the running coupling constant $\alpha\left(p^{2}\right)$ could be investigated by looking at the form factors of the gluon and ghost propagators. Such lattice simulations and studies based on the Schwinger-Dyson equations have provided evidence of the existence of an infrared fixed point in the Landau gauge for a renormalization group invariant coupling constant based on the gluon and ghost propagators (see e.g. [24]), i.e.

$$
\alpha(0) \approx \frac{8.92}{N}
$$

a result that has received some numerical confirmation too, see e.g. [25].

Analogous relationships can be derived in the Coulomb gauge. A nice updated work on the status of the running coupling constant in the Coulomb gauge and its relationship with the Landau gauge can be found in [26].

Interestingly, it turns out that also in the MAG, the renormalization of the gauge coupling is related to that of the fields, according to

$$
Z_{g}=Z_{A_{\text {diag }}}^{-1 / 2}
$$

where $Z_{A_{\text {diag }}}$ is the renormalization factor of the diagonal component of the gauge field $[18,19,27]$. Analogously to the Landau gauge, this relationship suggests that the infrared behavior of the gauge coupling might be investigated by looking at the diagonal gluon propagator. 


\section{E. Lattice data in the MAG}

Lattice simulations of the MAG have given strong indications that the off-diagonal gluons acquire a relatively large mass. Let us mention here that the MAG condition (13) amounts to keep as minimally as possible the off-diagonal components of the gauge fields. As a consequence, one expects that the Abelian components will play a predominant role. This is precisely the idea which underlies the Abelian dominance hypothesis.

The first study of the gluon propagator on the lattice in the maximal Abelian gauge was made in [15] in the case of $S U(2)$. The gluon propagator was analyzed in coordinate space. The off-diagonal component of the gluon propagator was found to be short-ranged, exhibiting a Yukawa type behavior

$$
G_{\mathrm{off}} \sim \frac{e^{-M_{\mathrm{off}} r}}{r^{3 / 2}} \text { with } M_{\mathrm{off}} \sim 1.2 \mathrm{GeV}
$$

The diagonal propagator was found to propagate over larger distances.

More recently, a numerical investigation of the gluon propagator in the maximal Abelian gauge has been worked out in [16]. Here, the gluon propagator was investigated in momentum space. At low momenta, the diagonal component of the gluon propagator has been found to be much larger than the off-diagonal one. In particular, a Gribov like fit,

$$
G_{\text {diag }}(q)=\frac{q^{2}}{q^{4}+m_{d g}^{4}},
$$

turns out to be suitable for the diagonal component of the gluon propagator. For off-diagonal gluons, a Yukawa type fit

$$
G_{\mathrm{off}}(q)=\frac{1}{q^{2}+M_{\mathrm{off}}^{2}},
$$

seems to work quite well. The mass parameter $M_{\text {off }}$ appearing in the Yukawa fit is two times bigger that the corresponding mass parameter $m_{\text {diag }}$ of the Gribov fit, namely

$$
M_{\text {off }} \approx 2 m_{\text {diag }},
$$

where $M_{\text {off }} \approx 1.2 \mathrm{GeV}$, in agreement with the result obtained in [15]. This implies that the off-diagonal propagator is shortranged as compared to the diagonal one, a fact which can serve as an indication for a kind of Abelian dominance.

We hope that it has become clear by now that the MAG is an important gauge, and deserves our attention.

\section{GRIBOV COPIES IN THE MAG AND RESTRICTION TO THE FIRST GRIBOV HORIZON}

The Gribov ambiguity is due to the fact that a gauge fixing should in principle select a single representative of a gauge orbit of any given gauge field configuration. Gribov has shown in the seminal paper [3] that, at least in the Landau and Coulomb gauge, there exist gauge equivalent field configurations obeying the Landau (or Coulomb) gauge. Gribov has worked out a method to restrict more tightly the domain of integration in the path integral for what considers the gauge fields.

Let us work out the condition for the existence of Gribov copies in the MAG. In the case of small gauge transformations, this is easily obtained by requiring that the gauge transformed fields (12), fulfill the same gauge conditions obeyed by $\left(A_{\mu}, A_{\mu}^{a}\right)$, i.e. (13) and (15). Thus, to the first order in the gauge parameters $\left(\omega, \omega^{a}\right)$, one gets

$$
\begin{aligned}
-D_{\mu}^{a b} D_{\mu}^{b c} \omega^{c}-g \varepsilon^{b c} D_{\mu}^{a b}\left(A_{\mu}^{c} \omega\right) & +g \varepsilon^{a b} A_{\mu}^{b} \partial_{\mu} \omega \\
+g^{2} \varepsilon^{a b} \varepsilon^{c d} A_{\mu}^{b} A_{\mu}^{c} \omega^{d} & =0, \\
-\partial^{2} \omega-g \varepsilon^{a b} \partial_{\mu}\left(A_{\mu}^{a} \omega^{b}\right) & =0,
\end{aligned}
$$

which, due to (13) and (15) simplify to

$$
\begin{aligned}
\mathcal{M}^{a b} \omega^{b} & =0, \\
-\partial^{2} \omega-g \varepsilon^{a b} \partial_{\mu}\left(A_{\mu}^{a} \omega^{b}\right) & =0,
\end{aligned}
$$

with

$$
\mathcal{M}^{a b}=-D_{\mu}^{a c} D_{\mu}^{c b}-g^{2} \varepsilon^{a c} \varepsilon^{b d} A_{\mu}^{c} A_{\mu}^{d} .
$$

This operator $\mathcal{M}^{a b}$ is recognized to be the Faddeev-Popov operator for the off-diagonal ghost sector, see [28, 29]. It enjoys the property of being Hermitian [30], thus having real eigenvalues.

One may thus expect the appearance of Gribov copies in the MAG. And indeed, a normalizable zero mode of the FaddeevPopov operator (30) was constructed in [30].

It was shown in [28] that the partition function for the MAG, described by the action (16) can be recast into the form

$$
Z=\int D A_{\mu} D A_{\mu}^{a} \delta\left(D_{\mu}^{a b} A_{\mu}^{b}\right) \delta\left(\partial_{\mu} A_{\mu}\right) \operatorname{det} \mathcal{M}^{a b} e^{-S_{\mathrm{YM}}},
$$

in the limit $\alpha \rightarrow 0$ and after integration over the Lagrange multipliers as well as over the off-diagonal and diagonal ghost fields, in the latter case a nontrivial field transformation was used.

Let us now briefly explain the idea of Gribov to restrict the domain of integration further [3], applied to the MAG. The interested reader can find the details for the MAG in [28]. We define the Gribov region $\mathcal{C}_{0}$ as the set of fields fulfilling the gauge conditions (13), (15) and for which the Faddeev-Popov operator $\mathcal{M}^{a b}$ is positive definite, namely

$$
C_{0}=\left\{A_{\mu}, A_{\mu}^{a}, \partial_{\mu} A_{\mu}=0, D_{\mu}^{a b} A_{\mu}^{b}=0, \mathcal{M}^{a b}>0\right\} .
$$

The boundary, $l_{1}$, of the region $C_{0}$, where the first vanishing eigenvalue of $\mathcal{M}^{a b}$ appears, is called the first Gribov horizon. The restriction of the domain of integration to this region is supported by the possibility of generalizing to the maximal Abelian gauge Gribov's original result [3] stating that for any field located near a horizon there is a gauge copy, close to the 
same horizon, located on the other side of the horizon. This statement for the MAG was explicitly proven in [28].

The idea of Gribov was now to restrict the domain of integration to the Gribov region. Therefore, we modify the MAG partition function (31) to

$Z=\int D A_{\mu}^{a} D A_{\mu} \operatorname{det} \mathcal{M}^{a b}(A) \delta\left(D_{\mu}^{a b} A_{\mu}^{b}\right) \delta\left(\partial_{\mu} A_{\mu}\right) e^{-S_{Y M}} \mathcal{V}\left(C_{0}\right)$,

where the factor $\mathcal{V}\left(\mathcal{C}_{0}\right)$ implements the restriction to the region $\mathcal{C}_{0}$. Following [3], the factor $\mathcal{V}\left(\mathcal{C}_{0}\right)$ can be accommodated for by means of a so-called "no pole condition" on the off-diagonal ghost two-point function, which is nothing else than the inverse of the Faddeev-Popov operator $\mathcal{M}^{a b}$. More precisely, denoting by $\mathcal{G}(k, A)$ the Fourier transform of $\left(\mathcal{M}^{a b}\right)^{-1}$, we shall require that $\mathcal{G}(k, A)$ has no poles for a given nonvanishing value of the momentum $k$, except for a singularity at $k=0$, corresponding to the boundary of $\mathcal{C}_{0}$, i.e. to the first Gribov horizon $l_{1}$ [3]. This no pole condition can be easily understood by observing that, within the region $\mathcal{C}_{0}$, the Faddeev-Popov operator $\mathcal{M}^{a b}$ is positive definite. This implies that its inverse, $\left(\mathcal{M}^{a b}\right)^{-1}$, and thus the Green function $\mathcal{G}(k, A)$, can become large only when approaching the horizon $l_{1}$, where the operator $\mathcal{M}^{a b}$ has a zero mode.

\section{A. Effects on the propagators}

The explicit implementation of the factor $\mathcal{V}\left(\mathcal{C}_{0}\right)$ can be found in [28]. Currently, we are more interested in the eventual implications on the propagators of the $S U(2)$ MAG theory.

For off-diagonal gluon propagator, it was found that

$$
\left\langle A_{\mu}^{a} A_{v}^{b}\right\rangle_{q}=\delta^{a b} \frac{1}{q^{2}}\left(\delta_{\mu v}-\frac{q_{\mu} q_{v}}{q^{2}}\right),
$$

while for the diagonal gluon propagator, it holds that

$$
\left\langle A_{\mu} A_{v}\right\rangle_{q}=\frac{q^{2}}{q^{4}+\gamma^{4}}\left(\delta_{\mu v}-\frac{q_{\mu} q_{v}}{q^{2}}\right) .
$$

We notice the appearance of the so-called Gribov mass parameter, which is the solution of the gap equation

$$
\frac{3}{4} g^{2} \int \frac{d^{4} p}{(2 \pi)^{4}} \frac{1}{p^{4}+\gamma^{4}}=1
$$

One sees that the diagonal component, (35), is suppressed in the infrared, exhibiting the characteristic Gribov type behavior. The off-diagonal components, (34), remains unchanged. However, as we shall soon see, its infrared behavior turns out to be modified once the gluon condensate $\left\langle A_{\mu}^{a} A_{\mu}^{a}\right\rangle$ is taken into account.

Concerning the ghost propagators, it can be shown that the diagonal ghost propagator is left unaffected by the restriction to the first Gribov region (see [28]). For the (trace of the) off-diagonal ghost propagator, one finds

$$
\mathcal{G}(q)_{q \approx 0} \approx \frac{\gamma^{2}}{q^{4}}
$$

exhibiting the typical infrared enhancement due to the Gribov restriction.

\section{DYNAMICAL OFF-DIAGONAL GLUON MASS}

In [19], the generation of the dimension two gluon condensate $\left\langle A_{\mu}^{a} A_{\mu}^{a}\right\rangle$ was discussed. A renormalizable effective potential for $\left\langle A_{\mu}^{a} A_{\mu}^{a}\right\rangle$ in the MAG has been constructed and evaluated in analytic form in [19]. A nonvanishing condensate $\left\langle A_{\mu}^{a} A_{\mu}^{a}\right\rangle$ is favoured since it lowers the vacuum energy. As a consequence, a dynamical tree level mass for off-diagonal gluons is generated. The combination of the condensate $\left\langle A_{\mu}^{a} A_{\mu}^{a}\right\rangle$ within the Gribov approximation can be performed along the lines outlined in [31], where the effects of the Gribov copies on the gluon and ghost propagators in the presence of the dimension two gluon condensate have been worked out in the Landau gauge. Following [19], the dynamical mass generation is accounted for by adding to the gauge fixed Yang-Mills action the following term [32]

$$
S_{\sigma}=\int d^{4} x\left(\frac{\sigma^{2}}{2 g^{2} \zeta}+\frac{1}{2} \frac{\sigma}{g \zeta} A_{\mu}^{a} A_{\mu}^{a}+\frac{1}{8 \zeta}\left(A_{\mu}^{a} A_{\mu}^{a}\right)^{2}\right) .
$$

The field $\sigma$ is an auxiliary field which allows one to study the condensation of the local operator $A_{\mu}^{a} A_{\mu}^{a}$, since [19]

$$
\langle\sigma\rangle=-\frac{g}{2}\left\langle A_{\mu}^{a} A_{\mu}^{a}\right\rangle \text {. }
$$

The dimensionless parameter $\zeta$ in expression (38) is needed to account for the ultraviolet divergences present in the vacuum correlation function $\left\langle A^{2}(x) A^{2}(y)\right\rangle$. For the details of the renormalizability properties of the local operator $A_{\mu}^{a} A_{\mu}^{a}$ in the maximal Abelian gauge we refer to [19] and references therein. The inclusion of the term $S_{\sigma}$ is the starting point for evaluating the renormalizable effective potential $V(\sigma)$ for the auxiliary field $\sigma$, which is moreover consistent with the renormalization group equations. The minimum of $V(\sigma)$ occurs for a nonvanishing vacuum expectation value $\sigma,\langle\sigma\rangle \neq 0$. In particular, the first order off-diagonal dynamical gluon mass turns out to be [19]

$$
m^{2} \equiv \frac{\langle\sigma\rangle}{g \zeta} \approx\left(2.25 \Lambda_{\overline{\mathrm{MS}}}\right)^{2}
$$

The inclusion of the action $S_{\sigma}$ leads to a partition function which is still plagued by the Gribov copies. It might be useful to note in fact that $S_{\sigma}$ is left invariant by the local gauge transformations (12), supplemented with

$$
\delta \sigma=g A_{\mu}^{a} D_{\mu}^{a b} \omega^{b} .
$$

The same procedure as before in the absence of $\left\langle A_{\mu}^{a} A_{\mu}^{a}\right\rangle$ can be repeated, and the outcome is that (35) and (37) are still valid, but the off-diagonal gluon propagator (34) gets modified to

$$
\left\langle A_{\mu}^{a} A_{v}^{b}\right\rangle_{q}=\delta^{a b} \frac{1}{q^{2}+m^{2}}\left(\delta_{\mu v}-\frac{q_{\mu} q_{v}}{q^{2}}\right)
$$

at lowest order. 


\section{COMPARISON WITH MAG LATTICE DATA}

Although the extrapolation of the lattice data in the region $q \approx 0$ is a difficult task, requiring large lattice volumes, the results (35) and (42) on the (transverse) diagonal and offdiagonal components of the gluon propagator can be considered to be in qualitative agreement with the lattice results, especially with the two parameter fits (23) and (24). Concerning now the ghost propagator, to our knowledge, no lattice data is available so far.

\section{CONCLUSION}

We have reviewed some analytically obtained results on the infrared behavior of the gluon and ghost propagators in the MAG. The diagonal gluon propagator displays a Gribov type behavior (35), while the off-diagonal one has a Yukawa type behavior (42), with an off-diagonal mass originating from the dimension two condensate $\left\langle A_{\mu}^{a} A_{\mu}^{a}\right\rangle$. These results are in satisfactory agreement with the available lattice data from $[15,16]$, summarized in (22), (23) and (24).

We hope these results might stimulate further investigation of Yang-Mills theories in the MAG. A first point of interest is the behaviour of the ghost propagators in the infrared, on which there is no lattice data available yet. A look at the offdiagonal ghost propagator from lattice simulations would be of a certain interest in order to improve our understanding of the influence of the Gribov copies in the MAG. A second point worth to be investigated is the all order relation (21), which could allow one to get information on the behavior of the gauge coupling constant in the infrared from the study of the diagonal component of the gluon propagator, allowing thus for a comparison with similar results obtained in Landau and Coulomb gauges.

\section{Acknowledgments}

S.P. Sorella would like to thank the "I LAWHEP"-organizers for this interesting conference. The Conselho Nacional de Desenvolvimento Científico e Tecnológico (CNPq-Brazil), the Faperj, Fundação de Amparo à Pesquisa do Estado do Rio de Janeiro, the SR2-UERJ and the Coordenação de Aperfeiçoamento de Pessoal de Nível Superior (CAPES) are gratefully acknowledged for financial support. D. Dudal is a postdoctoral fellow of the Special Research Fund of Ghent University.
[1] D. J. Gross and F. Wilczek, Phys. Rev. Lett. 30, 1343 (1973).

[2] H. D. Politzer, Phys. Rev. Lett. 30, 1346 (1973).

[3] V. N. Gribov, Nucl. Phys. B 139, 1 (1978).

[4] M. A. Shifman, A. I. Vainshtein, and V. I. Zakharov, Nucl. Phys. B 147, 519 (1979).

[5] F. V. Gubarev, L. Stodolsky, and V. I. Zakharov, Phys. Rev. Lett. 86, 2220 (2001).

[6] D. Dudal, H. Verschelde, J. A. Gracey, V. E. R. Lemes, M. S. Sarandy, R. F. Sobreiro, and S. P. Sorella, JHEP 0401, 044 (2004).

[7] T. Schafer and E. V. Shuryak, Rev. Mod. Phys. 70, 323 (1998).

[8] A. Maas, hep-th/0511307.

[9] G. 't Hooft, High Energy Physics EPS Int. Conference, Palermo 1975, ed. A. Zichichi.

[10] Y. Nambu, Phys. Rev. D 10, 4262 (1974).

[11] S. Mandelstam, Phys. Rept. 23, 245 (1976).

[12] G. 't Hooft, Nucl. Phys. B 190, 455 (1981).

[13] A. S. Kronfeld, M. L. Laursen, G. Schierholz, and U. J. Wiese, Phys. Lett. B 198, 516 (1987).

[14] A. S. Kronfeld, G. Schierholz, and U. J. Wiese, Nucl. Phys. B 293, 461 (1987).

[15] K. Amemiya and H. Suganuma, Phys. Rev. D 60, 114509 (1999).

[16] V. G. Bornyakov, M. N. Chernodub, F. V. Gubarev, S. M. Morozov, and M. I. Polikarpov, Phys. Lett. B 559, 214 (2003).

[17] H. Min, T. Lee, and P. Y. Pac, Phys. Rev. D 32, 440 (1985).

[18] A. R. Fazio, V. E. R. Lemes, M. S. Sarandy, and S. P. Sorella, Phys. Rev. D 64, 085003 (2001).
[19] D. Dudal, J. A. Gracey, V. E. R. Lemes, M. S. Sarandy, R. F. Sobreiro, S. P. Sorella, and H. Verschelde, Phys. Rev. D 70, 114038 (2004).

[20] Z. F. Ezawa and A. Iwazaki, Phys. Rev. D 25, 2681 (1982).

[21] T. Suzuki and I. Yotsuyanagi, Phys. Rev. D 42, 4257 (1990).

[22] S. Hioki, S. Kitahara, S. Kiura, Y. Matsubara, O. Miyamura, S. Ohno, and T. Suzuki, Phys. Lett. B 272, 326 (1991) [Erratum-ibid. B 281, 416 (1992)].

[23] O. Piguet and S. P. Sorella, Lect. Notes Phys. M28, 1 (1995).

[24] C. Lerche and L. von Smekal, Phys. Rev. D 65, 125006 (2002).

[25] J. C. R. Bloch, A. Cucchieri, K. Langfeld, and T. Mendes, Nucl. Phys. B 687, 76 (2004).

[26] C. S. Fischer and D. Zwanziger, Phys. Rev. D 72, 054005 (2005).

[27] J. A. Gracey, JHEP 0504, 012 (2005).

[28] M. A. L. Capri, V. E. R. Lemes, R. F. Sobreiro, S. P. Sorella, and R. Thibes, Phys. Rev. D 72, 085021 (2005).

[29] M. Quandt and H. Reinhardt, Int. J. Mod. Phys. A 13, 4049 (1998).

[30] F. Bruckmann, T. Heinzl, A. Wipf, and T. Tok, Nucl. Phys. B 584, 589 (2000)

[31] R. F. Sobreiro, S. P. Sorella, D. Dudal, and H. Verschelde, Phys. Lett. B 590, 265 (2004).

[32] We recall here that in principle, one has to employ the renormalizable action (16). As a consequence, the slightly more general operator $\frac{1}{2} A_{\mu}^{a} A_{\mu}^{a}+\alpha \bar{c}^{a} c^{a}$ has to used. At the end, one can consider the limit $\alpha \rightarrow 0$. We refer to [19] for more details. 